\title{
PKM2 and cancer: The function of PKM2 beyond glycolysis (Review)
}

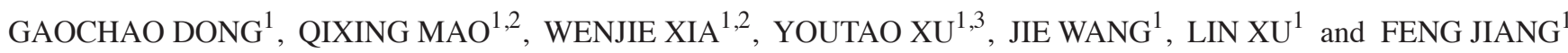 \\ ${ }^{1}$ Department of Thoracic Surgery, Nanjing Medical University Affiliated Cancer Hospital, \\ Jiangsu Key Laboratory of Molecular and Translational Cancer Research, Cancer Institute of Jiangsu Province, \\ Nanjing, Jiangsu 210009; ${ }^{2}$ The Fourth Clinical College of Nanjing Medical University; \\ ${ }^{3}$ The First Clinical College of Nanjing Medical University, Nanjing, Jiangsu 210000, P.R. China
}

Received February 23, 2015; Accepted January 18, 2016

DOI: $10.3892 / 01.2016 .4168$

\begin{abstract}
Metabolic reprogramming is a hallmark of cancer cells and is used by cancer cells for growth and survival. Pyruvate kinase muscle isozyme M2 (PKM2) is a limiting glycolytic enzyme that catalyzes the final step in glycolysis, which is key in tumor metabolism and growth. The present review discusses the expression and regulation of PKM2, and reports the dominant role that PKM2 plays in glycolysis to achieve the nutrient demands of cancer cell proliferation. In addition, the present study discusses the non-metabolic function of PKM2, and its role as a coactivator and protein kinase, which contributes to tumorigenesis. Furthermore, conflicting studies concerning the role of PKM2 as a therapeutic target are reviewed. The improved understanding of PKM2 may provide a noval approach for cancer treatment.
\end{abstract}

\section{Contents}

1. Introduction

2. The PKM2 gene and its regulation

3. Active and inactive oligomeric forms of PKM2: Activity regulation

Correspondence to: Professor Lin Xu or Dr Feng Jiang, Department of Thoracic Surgery, Nanjing Medical University Affiliated Cancer Hospital, Jiangsu Key Laboratory of Molecular and Translational Cancer Research, Cancer Institute of Jiangsu Province, 42 Baiziting, Nanjing, Jiangsu 210009, P.R. China

E-mail: xulin83cn@gmail.com

E-mail: zengnljf@hotmail.com

Abbreviations: PKM2, pyruvate kinase muscle isozyme M2; ATP, adenosine triphosphate; ROS, reactive oxygen species; EGFR, epidermal growth factor receptor; PEP, phosphoenolpyruvate; mTOR, mammalian target of rapamycin; HIF-1 $\alpha$, hypoxia inducible factor- $1 \alpha$; NF- $\kappa B$, nuclear factor $\kappa$ enhancer binding protein; PKC, protein kinase $\mathrm{C}$; IKK, IкB kinase; FBP, fructose-1,6-bisphosphate

Key words: PKM2, glycolysis, cancer, metabolic reprogramming, Warburg effect, novel function
4. Non-metabolic functions of PKM2

5. PKM2 as a therapeutic target

6. Conclusion

\section{Introduction}

In the 1920s, Warburg observed that cancer cells had a distinct metabolism that was highly dependent on glycolysis instead of mitochondrial oxidative phosphorylation, regardless of oxygen availability (1). This process was termed the Warburg effect, or aerobic glycolysis (2). Hanahan and Weinberg (3) also revealed that reprogramming energy metabolism was one of the most common characteristics of cancer cells. Compared with oxidative phosphorylation, glycolysis is a less efficient pathway for producing adenosine triphosphate (ATP) (4). To compensate for the loss of ATP due to preferential glycolysis, cancer cells upregulate genes that encode glucose transporters and glycolytic enzymes, which leads to glucose uptake and an altered metabolism (5). Increased glucose uptake is used in the clinic for the detection of tumors using fluorodeoxyglucose positron emission tomography (4). The metabolic reprogramming of cancer cells provides ATP and allows cells to survive under hypoxic conditions (6). In addition, metabolic reprogramming provides cells with biosynthetic building blocks, including intermediates and substrates for the synthesis of nucleotides, proteins and membrane components, which are required in proliferating cells (7).

Pyruvate kinase $(\mathrm{PK})$ is a rate-limiting glycolytic enzyme that catalyzes the irreversible transphosphorylation between phosphoenolpyruvate (PEP) and adenosine diphosphate, which produces pyruvate and ATP (8). This reaction is a committed step that leads to glycolysis or oxidative phosphorylation of pyruvate (Fig. 1). In mammals, the PK family consists of four isoforms: liver-type PK (PKL); red blood cell PK (PKR); and PK muscle isozyme M1 and M2 (PKM1 and PKM2, respectively). These isoforms are encoded by two genes, PK, liver and red blood cell (PKLR) and PK, muscle (PKM) $(9,10)$. The expression of pyruvates is tissue-specific and regulated by various promoters and alternative splicing. PKL and PKR are products of the PKLR gene. PKL is expressed in the liver, kidneys and intestine and has the lowest affinity to PEP 
of the isoforms, while PKR is expressed in red blood cells, inhibited by ATP and activated by fructose-1,6-bisphosphate (FBP). PKM1 and PKM2 are produced by alternative splicing of the primary RNA transcript of the PKM gene, which contains sequences encoded by exons 9 and 10, respectively. The non-allosteric isoform PKM1 is constitutively active, and expressed in terminally differentiated tissues, including the muscle and brain, which require a large supply of ATP. By contrast, PKM2 is expressed in tissues with anabolic functions, including proliferating cells and cancer cells, and is subject to complex allosteric regulation. In the majority of cancer cells, the expression of PKM2 is increased, which suggests that PKM2 may be an attractive target for cancer therapy (11). The identification of a novel protein that interacts with PKM2 has confirmed the key function of PKM2 in tumor metabolism and cancer growth (12), revealing novel functions of PKM2 beyond the classical concept of glycolysis.

\section{The PKM2 gene and its regulation}

The PKM2 gene is expressed by alternative splicing of PKM pre-messenger (m)RNA (13). In cancer cells, PKM2 is overexpressed, and overexpression is controlled by the oncoprotein c-Myc. c-Myc activates the transcription of heterogeneous nuclear ribonucleoproteins (hnRNPs) I, A1 and A2, which bind and repress exon 9 encoding RNA sequences (13). This results in the inhibition of PKM1 mRNA splicing, which allows the synchronous expression of the PKM2 isoform (13). A previous study demonstrated that PKM was regulated by the reciprocal effects of the mutually exclusive exons 9 and 10, which lead to the repression of exon 9 and the activation of exon 10 in cancer cells (14). In addition, knockdown of the serine/arginine-rich family of pre-mRNA splicing factor, which reduces lactate production, triggers the expression of exon 10 and promotes the proliferation of cells and aerobic glycolysis. This study suggested that the exonic elements are the actual determinants of PKM2 splicing, as opposed to classical intronic regions.

In the last decade, studies have focused on the metabolism and signal transduction of cells, and have revealed that oncogenes may have direct or indirect associations with metabolic reprogramming $(15,16)$. Mammalian target of rapamycin (mTOR) was demonstrated to be a key activator of the Warbug effect, as it induces PKM2 and other glycolytic enzymes under normoxic conditions (17). Sun et al (17) demonstrated that PKM2 was a crucial glycolytic enzyme in the oncogenic mTOR-induced Warbug effect, in which hypoxia inducible factor- $1 \alpha$ (HIF-1 $\alpha)$ and c-Myc-hnRNP cascades are the transducers of mTOR regulation of PKM2. Additionally, insulin is closely associated with cancer progression, and also upregulates PKM2 expression through phosphatidylinositide 3 -kinase/mTOR mediated HIF-1 $\alpha$ induction (18). Notably, the reduction in the activity of PKM2 is independent of this pathway; insulin-induced reactive oxygen species (ROS) was revealed to be responsible (18). Under hypoxic conditions, the PKM2 gene interacts directly with HIF-1 $\alpha$, which activates the hypoxia response element that is required for HIF-1 $\alpha$ binding (19).

A previous study demonstrated that the transcription of PKM2 was upregulated by the epidermal growth factor receptor (EGFR) under normoxic conditions (20). EGFR activation induces phospholipase $\mathrm{C} \gamma 1$-dependent protein kinase $\mathrm{C}(\mathrm{PKC}) \varepsilon$ monoubiquitination at lysine-321, which is mediated by RING-finger protein that interacts with $\mathrm{C}$ kinase 1 (21,22). Monoubiquitinated PKC $\varepsilon$ interacts with an ubiquitin-binding domain in the zinc finger domain of $\mathrm{I} \kappa \mathrm{B}$ kinase (IKK) $\gamma$, which leads to the recruitment of cytosolic IKK to the plasma membrane. PKC $\varepsilon$ phosphorylates IKK $\beta$ at serine-177, which activates IKK $\beta$. Activated v-rel avian reticuloendotheliosis viral oncogene homolog A (RelA) interacts with HIF-1 $\alpha$, which is required for the PKM promoter to bind to RelA $(21,22)$. EGFR-promoted glycolysis and tumorigenesis requires $\mathrm{PKC} \varepsilon$ - and nuclear factor $\kappa$ enhancer binding protein $(\mathrm{NF} \kappa \mathrm{B})$-dependent PKM2 upregulation $(21,22)$. These molecular interactions reveal the importance of the association between EGFR and $\mathrm{NF \kappa B}$ pathways in the upregulation of PKM2 and tumorigenesis of cells.

\section{Active and inactive oligomeric forms of PKM2: Activity regulation}

Cells have evolved complex regulatory mechanisms to adapt the metabolism to various physiological states. Rapidly growing cells consume nutrients at a high rate and must maintain a balance between the utilization of nutrients for ATP synthesis and anabolic development, including protein, lipid and nucleic acid synthesis. Cancer cells use glucose at higher rates compared to non-cancerous cells, but use a smaller fraction for oxidative phosphorylation, which enables cancer cells to incorporate a greater fraction of glucose metabolites in macromolecule synthesis instead of expending it on carbon dioxide production $(2,3)$. Consequently, metabolic programming of cancer cells is required to be flexible, which allows the cells to adapt to various environmental conditions. PKM2 is responsible for the final step of glycolysis and is key in this process $(2,3)$. The preferential expression and allosteric enzymatic activity of PKM2 provides the cancer cells with a growth advantage in vivo, without the accumulation of ROS.

Allosteric regulation of PKM2 allows switching between a high- and low-activity state. PKM2 exists in the catalytically distinct tetrameric and dimeric states $(2,3)$. Tetrameric PKM2 exhibits high catalytic activity, which is associated with ATP synthesis and catabolic metabolism in cells. Dimeric PKM2 has low catalytic activity and is the less active state of PKM2 that facilitates the production of glycolytic intermediates to enter the glycolysis branch pathways, including glycerol synthesis and the pentose phosphate pathway, which produces nicotinamide adenine dinucleotide phosphate-oxidase (NADPH) to suppress ROS production and is involved in nucleotide synthesis $(2,3)$.

Various factors have been reported that control the alteration between the dimer and tetramer of PKM2 (Fig. 2) (23). FBP is an upstream glycolytic intermediate and is a well-characterized activator of PKM2, which binds allosterically to PKM2 and facilitates the formation of the active tetramer (11). In addition, serine, which is a glycolytic intermediate 3-phosphoglycerate, is a positive regulator of PKM2 (24). Serine binds and activates PKM2, and a decrease in serine leads to a reduction in the PKM2 activity in cells (24). Phosphoribosylaminoimidazolesuccinocarboxamide (SAICAR) is an intermediate of the de novo purine nucleotide 


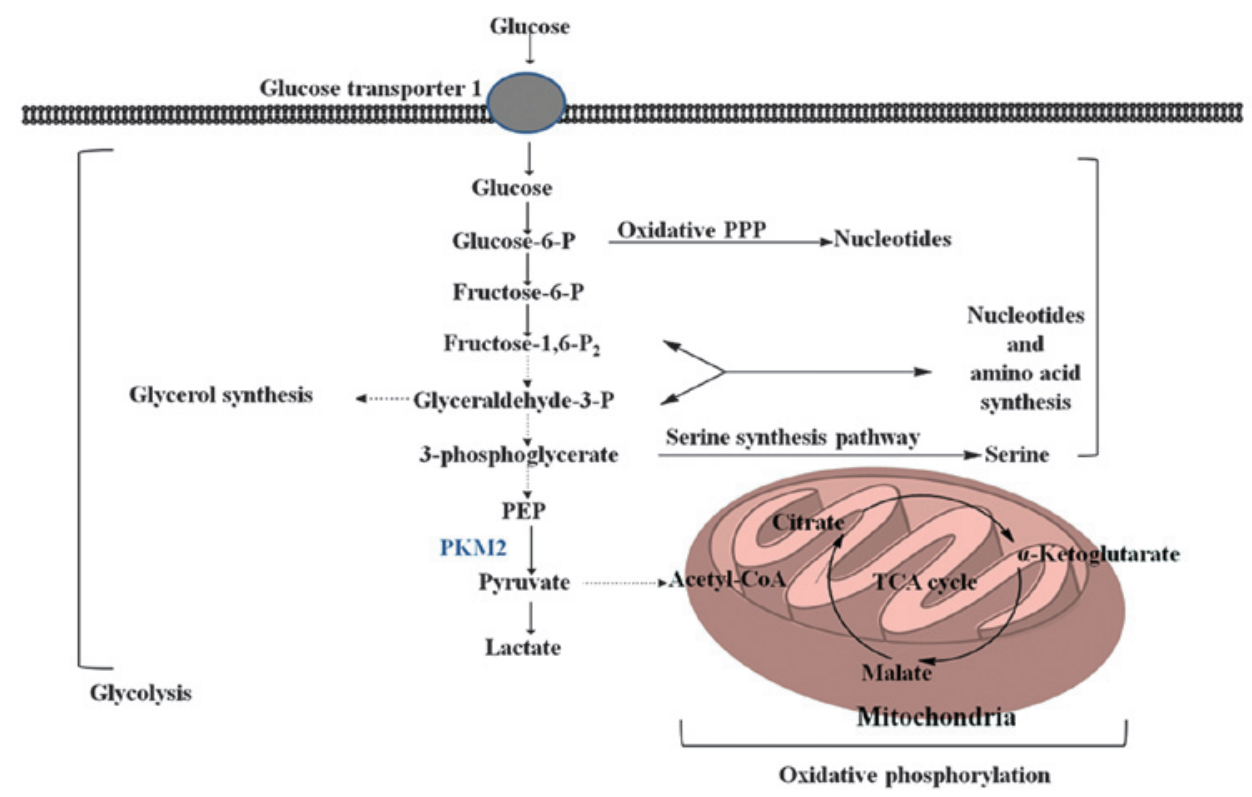

Figure 1. Metabolic pathway regulated by PKM2 in cancer cells. PKM2 is a less active isoform of the terminal glycolytic enzyme pyruvate kinase. The decreased enzymatic activity of PKM2 in the cytoplasm promotes the accumulation of upstream glycolytic intermediates and converts them into anabolic pathways. PKM2, pyruvate kinase muscle isozyme M2; P, phosphate; PEP, phosphoenolpyruvate; CoA, Coenzyme A; TCA, tricarboxylic acid; PPP, pentose phosphate pathway.

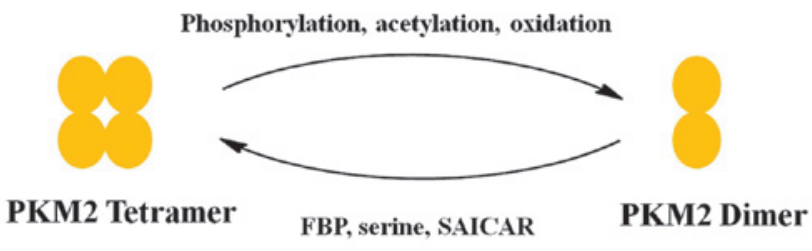

Figure 2. Allosteric regulation of PKM2 allows switching between a high- and low-activity state. The less active dimeric state of PKM2 is derived through phosphorylation, acetylation and oxidation of the PKM2 tetramer. The high-activity tetrameric state promotes the conversion of pyruvate to acetyl-Coenzyme A, which is stimulated by FBP, serine and SAICAR. Nutrient stress and growth factor signals also affect the state of PKM2. PKM2, pyruvate kinase muscle isozyme M2; FBP, fructose-1,6-bisphosphate; SAICAR, phosphoribosylaminoimidazolesuccinocarboxamide.

synthesis pathway and also stimulates PKM2 activity (25). SAICAR-PKM2 interaction promotes cancer cell survival in glucose-limited conditions (25-27). This allosteric regulation may lead to cancer cells coordinating various metabolic pathways to optimize cell growth in nutrient-limited conditions. PKM2 activity is also regulated by post-translational modifications, including phosphorylation, acetylation and oxidation, which favor the inactive dimeric state of PKM2 (25-27). Phosphorylation of PKM2 at tyrosine-105 induces the release of FBP, which causes PKM2 to alter between the tetrameric and dimeric states. Additionally, acetylation of PKM2 at lysine-305 downregulates PKM2 activity, which promotes glycolytic pooling, NADPH synthesis and tumor growth (25-27). Notably, a high glucose concentration induces a lysine-305 acetylation of PKM2, which diverts glucose to anabolic synthesis. Oxidation of PKM2 at cysteine-358 confers an advantage to cancer cells, as it allows the cells to withstand ROS (28). Intracellular ROS-induced oxidation of cysteine-358 decreases the activity of PKM2, which diverts glucose flux to the anabolic pentose phosphate pathway, thereby producing sufficient reducing potential for the detoxification of ROS.

Missense mutations in the PKM2 gene, including H391Y and K422R, are observed within the inter-subunit contact domain of the PKM2 protein, which may promote cancer metabolism, oxidative endurance, anchorage independence and tumor growth in a dominant-negative manner (29). The two gene mutations have different effects on the activity of PKM2, which does not affect the stability or expression level of the PKM2 protein. The H391Y mutated isoform of PKM2, resulting from a signal amino acid residue replacement, leads to the total loss of allosteric behavior, which affects the dynamic movement of the protein and results in cell rigidity (29).

\section{Non-metabolic functions of PKM2}

PKM2 acts as a coactivator. PKM2 is not only present in the cytoplasm of cells as a PK enzyme, but also translocates to the nucleus, which suggests PKM2 has functions in addition to glycolysis $(19,30)$. There has been a resurgence of interest in the metabolic effects of PKM2, and there have been recent studies revealing non-metabolic functions of PKM2. It has been reported that PKM2 translocates into the nucleus and interacts directly with the HIF-1 $\alpha$ subunit and promotes transactivation of HIF-1 target genes by enhancing HIF-1 binding and recruitment of p300, a transcriptional coactivator, which regulates HIF-1 activity $(19,30)$. PKM2 gene transcription is also activated by HIF-1, which creates a positive feedback loop that promotes HIF-1 transactivation and alters glucose metabolism in cancer cells (19). The Jumonji C domain-containing dioxygenase Jumonji domain-containing protein 5 (JMJD5) directly interacts with PKM2, which induces the translocation of PKM2 into the nucleus and promotes HIF-1 $\alpha$ mediated transactivation (31). The JMJD5-PKM2 interaction occurs 
at the intersubunit interface region of PKM2, which hinders PKM2 tetramerization and blocks PK activity.

The translocation of PKM2, and not PKM1, may be in response to EGFR activation (32). In PKM2, lysine-433 binds to $\mathrm{c}$-Src-phosphorylated tyrosine-333 on $\beta$-catenin, and this interaction is required for the two proteins to be recruited to the cyclin D1 (CCND1) promoter (32). This results in the removal of histone deacetylase 3 (HDAC3) from the promoter, histone H3 acetylation and CCND1 expression (12). PKM2-dependent $\beta$-catenin transactivation is required for EGFR-promoted tumor cell proliferation and brain tumor development (33). In addition, associations have been identified between $\mathrm{c}-\mathrm{Src}$ activity, $\beta$-catenin tyrosine-333 phosphorylation and PKM2 nuclear accumulation in human glioblastoma specimens (32). In colorectal cancer, decrease in the expression of PKM2 stimulates the $\beta$-catenin signaling pathway, which leads to the promotion of c-Myc-mediated glutamine metabolism (34). PKM2 may also be acetylated by p300 acetyltransferase at lysine-433, which prevents PKM2 activation by altering FBP (35). Activation of PKM2 promotes the nuclear accumulation and protein kinase activity, which is increased by cell cycle stimulation, EGF and oncoprotein E7. Phosphorylation of PKM2 at serine-37 promotes the translocation of PKM2 to the nucleus through the mitogen-activated protein kinase 1 ERK2 docking groove, which binds directly to PKM2 at isoleucine-429/leucine-431, and the protein kinase activity of PKM2 (36). Phosphorylation of PKM2 at serine-37 recruits peptidyl-prolyl cis-trans isomerase NIMA-interacting 1 for the cis-trans isomerization of PKM2 (36). In addition, nuclear PKM2 acts as a coactivator of $\beta$-catenin to induce the expression of c-Myc. Upregulation of c-Myc expression by PKM2 creates a positive feedback-loop, since c-Myc elevates the transcription of hnRNPs, which contributes to an increase in the PKM2/PKM1 ratio and promotes glycolysis by driving the expression of enzymatic kinases (36).

Protein kinase activity of PKM2. PKM2 not only acts as a coactivator, but may also act as a protein kinase that phosphorylates substrates involved in metabolic reprogramming. Nuclear dimeric PKM2 directly phosphorylates signal transducer and activator of transcription 3 (STAT3), which is activated in response to inflammatory cytokines, including interleukin-6 (37). PKM2 uses PEP as a phosphate donor to phosphorylate STAT3 at tyrosine-705, which activates the transcription of mitogen-activated protein kinase kinase 5. The activation of STAT3 in malignant cancer cells is possibly one of the most important molecular signatures for promoting the progression of cancer. PKM2 overexpression facilitates STAT3 nuclear translocation, which regulates the aggressive progression of colorectal cancer (38). The functional implications of the study by Yang et al (38) indicate that PKM2 activates $\beta 1$-integrin-focal adhesion kinase and snail-2-E-cadherin signaling, and also upregulates the expression of matrix metalloproteinase-2 and 9, which induces cell migration and adhesion. Similarly to STAT3, histone H3 is a substrate for PKM2 kinase activity (39). PKM2 directly binds and phosphorylates histone $\mathrm{H} 3$ at threonine-11, which leads to the removal of HDAC3 from CCND1 and Myc promoter regions, subsequent to acetylation at lysine-9 and gene transcription (39). PKM2-dependent histone H3 modifications are key to EGF stimulation (19). Furthermore, nuclear PKM2 levels are associated with phosphorylation at proline-3/threonine-11 in malignant glioma (36). Recently, a study reported that the PKM2-SAICAR interaction is required and sufficient to induce robust protein kinase activity in PKM2 in vitro and in cancer cells (26). It was also reported that the PKM2-SAICAR complex phosphorylates $>100$ human proteins, the majority of which were previously unrecognized. In particular, PKM2-SAICAR directly activates ERK1 in vitro; however, activated ERK1/2 phosphorylates PKM2, creating a positive feedback loop (36). Additionally, when EGFR is activated, the concentration of cellular SAICAR is increased, which is required to sustain the activation of ERK1/2 and proliferative signaling via PKM2 (36).

PKM2 acts as a differentiating agent. PKM2 acts as a proliferative agent through its interaction with nuclear proteins, and as a differentiating agent through its interaction with octamer-binding transcription factor 4 (Oct4), which is a key regulator in cancer stem cell self-renewal and differentiation (40). In glioma stem cells, the interaction between PKM2 and Oct4 inhibits the ability of PKM2 to maintain cell stemness, thereby promoting cell differentiation and enhancing the sensitivity of cells to cell death, which is concomitant with an alteration between the dimer and tetramer states of PKM2 (40). Notably, dichloroacetate, which is known as an inhibitor of PDK1 and is involved in the mitochondrial tricarboxylic acid cycle, increases the number of PKM2/Oct4 complexes (41).

PKM2 emergence in blood circulation. PKM2 detected in the blood of patients with gastrointestinal, pancreatic, lung and ovarian cancer and renal cell carcinoma revealed that PKM2 is released into the circulation (42). The PKM2 levels in the circulation of patients may be used as a diagnostic marker for numerous types of cancer (43). A recent study suggested that PKM2 in the blood circulation facilitated tumor growth by promoting tumor angiogenesis (44). The study demonstrated that PKM2 promoted tumor angiogenesis by increasing endothelial cell proliferation, migration and extracellular matrix-cell adhesions; only dimeric PKM2 was observed to be involved in tumor angiogenesis.

\section{PKM2 as a therapeutic target}

Cancer-specific metabolism, which is closely associated with tumorigenicity, cancer aggressive progression and inherent or acquired therapeutic resistance, is an attractive target for cancer therapy. The role of PKM2 in glycolysis and the proliferation of cancer cells has been revealed, and has been demonstrated to balance the production of biomolecular building blocks and the generation of pyruvate and ATP. Consequently, there are two therapeutic strategies for targeting PKM2. The first is inhibitors that block the catalytic activity of PKM2, and the second is activators that induce tetramerization of PKM2 to increase glycolysis.

Inhibition of PMK2 activity. Inhibitors of PKM2 appear to rely on the hypothesis that proliferating cells are highly dependent on energy, and a reduced activity of PKM2 inhibits energy 
regeneration. Using a high throughput screen, small molecules have been identified that selectively inhibit PKM2 (45). These molecules target the allosteric FBP binding site of PKM2; however, since PKL and PKR are also activated by FBP, there may be toxicity issues for human cancer therapy if PK activity is inhibited in the liver and red blood cells. Natural products, such as shikonin and its analog alkannin, which belong to a family of necroptotic inducers, demonstrated potent and promising selective inhibition of PKM2 that did not inhibit PKM1 and PKL (46). However, PKM2 inhibition by these drugs is partially reversed by the addition of FBP to protein lysates.

RNA interference targeting PKM2 has been observed to attenuate growth and induce caspase-dependent apoptosis in several cancer cell lines (47). In addition, peptide aptamers, which inhibit PKM2 and not the homologous PKM1, induce a significant decrease in cell proliferation and size under conditions where glucose metabolism is disrupted (48).

Although the inhibition of PKM2 has been demonstrated to be successful against cancer cell proliferation, the majority of cells are characterized by a high glutaminolysis capacity, which is another source of energy (47). Therefore, an inhibition of PKM2 may not be sufficient to significantly reduce the cellular proliferation of tumor cells.

Activators of PKM2. PKM2 activators aim to induce tetramerization of PKM2, which results in a decrease in glycolytic intermediates that are used as biosynthetic precursors. A class of quinolone sulfonamide activators has been reported to possess a distinctive mode of binding to PKM2 (49). These activators bind to a site that is distinct from the FBP binding site, which results in the diversion of glycolytic intermediates away from the serine biosynthetic pathway, which produces serine that is required for continued cell proliferation. A library of 13,000,000 drug-like compounds was screened in silico against the PKM2 structure, and 9 novel scaffolds were identified as PKM2 activators (50). These molecules had low nanomolar PKM2 potency, with excellent selectivity to other PK isoforms and good absorption, distribution, metabolism and excretion properties. A series of pyridopyrimidine analogs were also reported as potent activators of PKM2, with good stability, permeability, solubility and selectivity to PKM2 (51). However, PKM2 activation alone is not sufficient to alter cancer cell metabolism. Another series of small molecule PKM2 activators did not affect the growth of cancer cell lines under normal conditions in vitro, but strongly inhibited the proliferation of multiple lung cancer cell lines when serine was removed from the cell culture media (52). Screening of an in-house fragment-like library of 2,000 other compounds, led to the identification of another series of small molecules, which caused $50 \%$ of the cell population to undergo apoptosis at a concentration of $36 \mu \mathrm{M}$, with $104 \%$ maximal response compared with the natural PKM2 ligand and FBP activator (53). All the reported small molecule activators of PKM2 bind to a site that is distinct from the binding site of FBP, which is located on the opposite side of the tetramer interface compared with the FBP site.

By contrast, oleanolic acid (OA) did not interact with PKM2, which affects the splicing of the PKM gene (54). Decreased expression of c-Myc-dependent hnRNA1, a subtype of hnRNPs, and mTOR inhibition were responsible for the OA-induced switch between PKM isoforms.

Overall, there are a number of PKM2 inhibitors and activators that are in preclinical development; however, to date, none have advanced to human clinical studies. The present study suggests that PKM2 activators may be a more promising therapeutic target for patients with cancer compared with PKM2 inhibitors. However, the serine auxotrophy induced by PKM2 activators may be reversed by multiple pathways, including de novo serine synthesis, conversion from glycine, catabolism from proteins and serine-containing phospholipids and absorption from the extracellular environment, which may present a challenge when investigating activators.

\section{Conclusion}

The present review focused on the PKM2 gene and its regulation, and emphasized the non-metabolic function of PKM2 and the development of PKM2 as a therapeutic target. In the last decade, extensive research has clarified the notable involvement of PKM2 in cancer through its canonical and non-canonical functions. This allosteric property of PKM2 balances cell growth and oxidative stress.

However, PKM2 has multiple roles, and the intracellular mechanisms induced by PKM2 are more complicated than previously hypothesized. Protein kinases are important regulators in numerous biological processes. Numerous human proteins have been identified as potential substrates for PKM2, the majority of which are involved in the regulation of cell proliferation. Additional studies concerning the mechanism of PKM2 substrate recognition may reveal a common mechanism between the proteins involved in cell-cycle regulation. It has been demonstrated that the cell microenvironment provides metabolic heterogeneity in cancer tissues, which may be caused by differences in the supply of nutrients and oxygen (55). Cancer cells are located at various distances from blood vessels, in which PKM2 may play a role. Certain studies suggest that overexpression of PKM2 may be a biomarker for specific types of cancer (56-58); however, this is controversial and additional studies are required for confirmation. The regulation of PKM2 requires additional clarification. Even by providing a functional readout of the oxidative and metabolic state of the cell, it is not fully clear how PKM2 alters the response of the cell to growth factor stimulation (59); however, the association between PKM2 and growth factor responses and oxidative stress responses indicates that this regulation is integrated. It is also contradictory that certain studies report that knockdown of PKM2 results in a modest inhibitory effect on in vitro proliferation of cancer cells and xenograft tumors without signs of inhibition, which indicates that PKM2 is not a requirement for tumor growth $(45,46)$.

In conclusion, PKM2 is essential in the metabolic reprogramming of cancer cells; however, cancer cells reprogram using several metabolic pathways to support cell growth. An improved understanding of metabolism transformation in early cancer cells and how this results in the development and progression of cancer may provide an understanding of the metabolic and non-metabolic functions of PKM2. Furthermore, targeting PKM2 as a treatment for cancer may significantly evolve over the next several years. 


\section{Acknowledgements}

The present study was supported by the Natural Science Foundation of Jiangsu Province (grant no. BK2012482), National Natural Science Foundation of China (grant no. 81472702) and Jiangsu Province Special Program of Medical Science (grant no. BL2012030).

\section{References}

1. Warburg O, Wind F and Negelein E: The metabolism of tumors in the body. J Gen Physiol 8: 519-530, 1927.

2. Warburg O: On the origin of cancer cells. Science 123: 309-314, 1956.

3. Hanahan D and Weinberg RA: Hallmarks of cancer: The next generation. Cell 144: 646-674, 2011.

4. Hsu PP and Sabatini DM: Cancer cell metabolism: Warburg and beyond. Cell 134: 703-707, 2008.

5. Gambhir SS: Molecular imaging of cancer with positron emission tomography. Nat Rev Cancer 2: 683-693, 2002.

6. Mathupala SP, Rempel A and Pedersen PL: Glucose catabolism in cancer cells: identification and characterization of a marked activation response of the type II hexokinase gene to hypoxic conditions. J Biol Chem 276: 43407-43412, 2001.

7. Vander Heiden MG, Cantley LC and Thompson CB: Understanding the Warburg effect: The metabolic requirements of cell proliferation. Science 324: 1029-1033, 2009.

8. Altenberg B and Greulich KO: Genes of glycolysis are ubiquitously overexpressed in 24 cancer classes. Genomics 84 1014-1020, 2004.

9. Noguchi T, Inoue H and Tanaka T: The M1- and M2-type isozymes of rat pyruvate kinase are produced from the same gene by alternative RNA splicing. J Biol Chem 261: 13807-13812, 1986.

10. Noguchi T, Yamada K, Inoue $H$, Matsuda $T$ and Tanaka $T$ : The $\mathrm{L}$ - and R-type isozymes of rat pyruvate kinase are produced from a single gene by use of different promoters. J Biol Chem 262: 14366-14371, 1987.

11. Christofk HR, Vander Heiden MG, Harris MH, Ramanathan A, Gerszten RE, Wei R, Fleming MD, Schreiber SL and Cantley LC: The M2 splice isoform of pyruvate kinase is important for cancer metabolism and tumour growth. Nature 452: 230-233, 2008.

12. Yang W and Lu Z: Nuclear PKM2 regulates the Warburg effect. Cell Cycle 12: 3154-3158, 2013.

13. David CJ, Chen M, Assanah M, Canoll P and Manley JL: HnRNP proteins controlled by c-Myc deregulate pyruvate kinase mRNA splicing in cancer. Nature 463: 364-368, 2010.

14. Wang Z, Chatterjee D, Jeon HY, Akerman M, Vander Heiden MG, Cantley LC and Krainer AR: Exon-centric regulation of pyruvate kinase $\mathrm{M}$ alternative splicing via mutually exclusive exons. J Mol Cell Biol 4: 79-87, 2012.

15. DeBerardinis RJ, Lum JJ, Hatzivassiliou G and Thompson CB: The biology of cancer: Metabolic reprogramming fuels cell growth and proliferation. Cell Metab 7: 11-20, 2008.

16. Semenza GL: HIF-1: Upstream and downstream of cancer metabolism. Curr Opin Genet Dev 20: 51-56, 2010.

17. Sun Q, Chen X, Ma J, Peng H, Wang F, Zha X, Wang Y, Jing Y, Yang H, Chen R, et al: Mammalian target of rapamycin up-regulation of pyruvate kinase isoenzyme type M2 is critical for aerobic glycolysis and tumor growth. Proc Natl Acad Sci USA 108: 4129-4134, 2011

18. Iqbal MA, Siddiqui FA, Gupta V, Chattopadhyay S, Gopinath P, Kumar B, Manvati S, Chaman N and Bamezai RN: Insulin enhances metabolic capacities of cancer cells by dual regulation of glycolytic enzyme pyruvate kinase M2. Mol Cancer 12: 72, 2013.

19. Luo W, Hu H, Chang R, Zhong J, Knabel M, O'Meally R, Cole RN, Pandey A and Semenza GL: Pyruvate kinase M2 is a PHD3-stimulated coactivator for hypoxia-inducible factor 1 Cell 145: 732-744, 2011

20. Yang W, Xia Y, Cao Y, Zheng Y, Bu W, Zhang L, You MJ, Koh MY, Cote G, Aldape K, et al: EGFR-induced and PKCE monoubiquitylation-dependent $\mathrm{NF}-\mathrm{\kappa B}$ activation upregulates PKM2 expression and promotes tumorigenesis. Mol Cell 48 771-784, 2012

21. Breitkreutz D, Braiman-Wiksman L, Daum N, Denning MF and Tennenbaum T: Protein kinase C family: On the crossroads of cell signaling in skin and tumor epithelium. J Canc Res Clin Oncol 133: 793-808, 2007.
22. Choi JH, Ryu SH and Suh PG: On/off-regulation of phospholipase C-gamma 1-mediated signal transduction. Adv Enzyme Regul 47: 104-116, 2007.

23. Anastasiou D, Yu Y, Israelsen WJ, Jiang JK, Boxer MB, Hong BS, Tempel W, Dimov S, Shen M, Jha A, et al: Pyruvate kinase M2 activators promote tetramer formation and suppress tumorigenesis. Nat Chem Biol 8: 839-847, 2012.

24. Chaneton B, Hillmann P, Zheng L, Martin AC, Maddocks OD, Chokkathukalam A, Coyle JE, Jankevics A, Holding FP, Vousden $\mathrm{KH}$, et al: Serine is a natural ligand and allosteric activator of pyruvate kinase M2. Nature 491: 458-462, 2012.

25. Keller KE, Tan IS and Lee YS: SAICAR stimulates pyruvate kinase isoform M2 and promotes cancer cell survival in glucose-limited conditions. Science 338: 1069-1072, 2012.

26. Keller KE, Doctor ZM, Dwyer ZW and Lee YS: SAICAR induces protein kinase activity of PKM2 that is necessary for sustained proliferative signaling of cancer cells. Mol Cell 53: 700-709, 2014

27. Gui DY, Lewis CA and Vander Heiden MG: Allosteric regulation of PKM2 allows cellular adaptation to different physiological states. Sci Signal 6: pe7, 2013.

28. Anastasiou D, Poulogiannis G, Asara JM, Boxer MB, Jiang JK, Shen M, Bellinger G, Sasaki AT, Locasale JW, Auld DS, et al: Inhibition of pyruvate kinase M2 by reactive oxygen species contributes to cellular antioxidant responses. Science 334 : 1278-1283, 2011

29. Iqbal MA, Siddiqui FA, Chaman N, Gupta V, Kumar B, Gopinath $\mathrm{P}$ and Bamezai RN: Missense mutations in pyruvate kinase M2 promote cancer metabolism, oxidative endurance, anchorage independence, and tumor growth in a dominant negative manner. J Biol Chem 289: 8098-8105, 2014.

30. Luo W and Semenza GL: Pyruvate kinase M2 regulates glucose metabolism by functioning as a coactivator for hypoxia-inducible factor 1 in cancer cells. Oncotarget 2: 551-556, 2011.

31. Wang HJ, Hsieh YJ, Cheng WC, Lin CP, Lin YS, Yang SF, Chen CC, Izumiya Y, Yu JS, Kung HJ and Wang WC: JMJD5 regulates PKM2 nuclear translocation and reprograms HIF-1 $\alpha$-mediated glucose metabolism. Proc Natl Acad Sci USA 111: 279-284, 2014.

32. Yang W, Xia Y, Ji H, Zheng Y, Liang J, Huang W, Gao X, Aldape $\mathrm{K}$ and Lu Z: Nuclear PKM2 regulates $\beta$-catenin transactivation upon EGFR activation. Nature 480: 118-122, 2011.

33. Wu H, Li Z, Yang P, Zhang L and Fan Y: PKM 2 depletion induces the compensation of glutaminolysis through $\beta$-catenin/c-Myc pathway in tumor cells. Cell Signal 26: 2397-2405, 2014.

34. Li Z, Li X, Wu S, Xue M and Chen W: Long non-coding RNA UCA 1 promotes glycolysis by upregulating hexokinase 2 through the mTOR-STAT3/microRNA143 pathway. Cancer Sci 105: 951-955, 2014.

35. Lv L, Xu YP, Zhao D, Li FL, Wang W, Sasaki N, Jiang Y, Zhou X, Li TT, Guan KL, et al: Mitogenic and oncogenic stimulation of K433 acetylation promotes PKM2 protein kinase activity and nuclear localization. Mol Cell 52: 340-352, 2013.

36. Yang W, Zheng Y, Xia Y, Ji H, Chen X, Guo F, Lyssiotis CA, Aldape K, Cantley LC and Lu Z: ERK1/2-dependent phosphorylation and nuclear translocation of PKM2 promotes the Warburg effect. Nat Cell Biol 14: 1295-1304, 2012.

37. Gao X, Wang H, Yang JJ, Liu X and Liu ZR: Pyruvate kinase M2 regulates gene transcription by acting as a protein kinase. Mol Cell 45: 598-609, 2012.

38. Yang P, Li Z, Fu R, Wu H and Li Z: Pyruvate kinase M2 facilitates colon cancer cell migration via the modulation of STAT3 signalling. Cell Signal 26: 1853-1862, 2014.

39. Yang W, Xia Y, Hawke D, Li X, Liang J, Xing D, Aldape K, Hunter T, Alfred Yung WK and Lu Z: PKM2 phosphorylates histone $\mathrm{H} 3$ and promotes gene transcription and tumorigenesis. Cell 150: 685-696, 2012.

40. Morfouace M, Lalier L, Oliver L, Cheray M, Pecqueur C, Cartron PF and Vallette FM: Control of glioma cell death and differentiation by PKM2-Oct4 interaction. Cell Death Dis 5: e1036, 2014.

41. Bonnet S, Archer SL, Allalunis-Turner J, Haromy A, Beaulieu C, Thompson R, Lee CT, Lopaschuk GD, Puttagunta L, Bonnet $\mathrm{S}$, et al: A mitochondria- $\mathrm{K}^{+}$channel axis is suppressed in cancer and its normalization promotes apoptosis and inhibits cancer growth. Cancer Cell 11: 37-51, 2007.

42. Weinberger R, Appel B, Stein A, Metz Y, Neheman A and Barak M: The pyruvate kinase isoenzyme M2 (Tu M2-PK) as a tumour marker for renal cell carcinoma. Eur J Cancer Care (Engl) 16: 333-337, 2007. 
43. Ahmed AS, Dew T, Lawton FG, Papadopoulos AJ, Devaja O, Raju KS and Sherwood RA: M2-PK as a novel marker in ovarian cancer. A prospective cohort study. Eur J Gynaecol Oncol 28: 83-88, 2007.

44. Li L, Zhang Y, Qiao J, Yang JJ and Liu ZR: Pyruvate kinase M2 in blood circulation facilitates tumor growth by promoting angiogenesis. J Biol Chem 289: 25812-25821, 2014.

45. Vander Heiden MG, Christofk HR, Schuman E, Subtelny AO, Sharfi H, Harlow EE, Xian J and Cantley LC: Identification of small molecule inhibitors of pyruvate kinase M2. Biochem Pharmacol 79: 1118-1124, 2010.

46. Chen J, Xie J, Jiang Z, Wang B, Wang Y and Hu X: Shikonin and its analogs inhibit cancer cell glycolysis by targeting tumor pyruvate kinase-M2. Oncogene 30: 4297-4306, 2011.

47. Goldberg MS and Sharp PA: Pyruvate kinase M2-specific siRNA induces apoptosis and tumor regression. J Exp Med 209: 217-224, 2012.

48. Spoden GA, Rostek U, Lechner S, Mitterberger M, Mazurek S and Zwerschke W: Pyruvate kinase isoenzyme M2 is a glycolytic sensor differentially regulating cell proliferation, cell size and apoptotic cell death dependent on glucose supply. Exp Cell Res 315: 2765-2774, 2009.

49. Kung C, Hixon J, Choe S, Marks K, Gross S, Murphy E, DeLaBarre B, Cianchetta G, Sethumadhavan S, Wang X, et al: Small molecule activation of PKM2 in cancer cells induces serine au xotrophy. Chem Biol 19: 1187-1198, 2012.

50. Yacovan A, Ozeri R, Kehat T, Mirilashvili S, Sherman D, Aizikovich A, Shitrit A, Ben-Zeev E, Schutz N, Bohana-Kashtan O, et al: 1-(sulfonyl)-5-(arylsulfonyl)indoline as activators of the tumor cell specific M2 isoform of pyruvate kinase. Bioorg Med Chem Lett 22: 6460-6468, 2012.
51. Guo C, Linton A, Jalaie M, Kephart S, Ornelas M, Pairish M, GreasleyS, Richardson P, Maegley K, Hickey M, et al: Discovery of 2-((1H-benzo[d]imidazol-1-yl)methyl)-4H-pyrido[1,2-a] pyrimidin-4-ones as novel PKM2 activators. Bioorg Med Chem Lett 23: 3358-3363, 2013.

52. Parnell KM, Foulks JM, Nix RN, Clifford A, Bullough J, Luo B, Senina A, Vollmer D, Liu J, McCarthy V, et al: Pharmacologic activation of PKM2 slows lung tumor xenograft growth. Mol Cancer Ther 12: 1453-1460, 2013.

53. Xu Y, Liu XH, Saunders M, Pearce S, Foulks JM, Parnell KM, Clifford A, Nix RN, Bullough J, Hendrickson TF, et al: Discovery of 3-(trifluoromethyl)-1H-pyrazole-5-carboxamide activators of the M2 isoform of pyruvate kinase (PKM2). Bioorg Med Chem Lett 24: 515-519, 2014

54. Liu J, Wu N, Ma L, Liu M, Liu G, Zhang Y and Lin X: Oleanolic acid suppresses aerobic glycolysis in cancer cells by switching pyruvate kinase type M isoforms. PLoS One 9: e91606, 2014

55. Vaupel P: Metabolic microenvironment of tumor cells: A key factor in malignant progression. Exp Oncol 32: 125-127, 2010.

56. Wong N, Yan J, Ojo D, De Melo J, Cutz JC and Tang D: Changes in PKM2 associate with prostate cancer progression. Cancer Invest 32: 330-338, 2014.

57. Zhan C, Shi Y, Lu C and Wang Q: Pyruvate kinase M2 is highly correlated with the differentiation and the prognosis of esophageal squamous cell cancer. Dis Esophagus 26: 746-753, 2013.

58. Zhou CF, Li XB, Sun H, Zhang B, Han YS, Jiang Y, Zhuang QL, Fang J and Wu GH: Pyruvate kinase type M2 is upregulated in colorectal cancer and promotes proliferation and migration of colon cancer cells. IUBMB Life 64: 775-782, 2012.

59. Warner SL, Carpenter KJ and Bearss DJ: Activators of PKM 2 in cancer metabolism. Future Med Chem 6: 1167-1178, 2014. 